\title{
Characterization of Sirtuin Inhibitors in Nematodes Expressing a Muscular Dystrophy Protein Reveals Muscle Cell and Behavioral Protection by Specific Sirtinol Analogues
}

\author{
Matthieu Y. Pasco, ${ }^{\dagger, \$}$, Dante Rotili,, Lucia Altucci, ${ }^{\perp}$ Francesca Farina, ${ }^{\dagger, *}$ Guy A. Rouleau, ${ }^{\S}$ Antonello Mai, ${ }^{*, \|}$ and \\ Christian Néri*,† \\ ${ }^{\dagger}$ INSERM, Laboratory of Neuronal Cell Biology and Pathology, Center for Psychiatry and Neurosciences U894, ${ }^{\star}$ Université Paris Descartes, \\ Equipe d'accueil 4059, 75014 Paris, France, ${ }^{\S}$ University of Montreal, Department of Medicine and the Center for Excellence in Neuromics, \\ Montreal, Québec, Canada H2L 4M1, "Pasteur Institute-Cenci-Bolognetti Foundation, Dipartimento di Chimica e Tecnologie del Farmaco, \\ Sapienza University of Rome, 00185 Rome, Italy, and ${ }^{\perp}$ Dipartimento di Patologia Generale, Facoltà di Medicina e Chirurgia, \\ Seconda Università di Napoli, Vico L. De Crecchio 7, 80138 Napoli, Italy
}

Received September 8, 2009

\begin{abstract}
In oculopharyngeal muscular dystrophy (OPMD), a disease caused by polyalanine expansion in the nuclear protein PABPN1, the genetic inhibition of sirtuins and treatment with sirtuin inhibitors protect from mutant PABPN1 toxicity in transgenic nematodes. Here, we tested the SIRT1/2 inhibitors 1-12, bearing different degrees of inhibition, for protection against mutant PABPN1 toxicity in Caenorhabditis elegans. Compounds 2, 4, and $\mathbf{1 1}$ were the most efficient, revealing a potential therapeutic application for muscle cell protection in OPMD.
\end{abstract}

\section{Introduction}

Sirtuins are $\mathrm{NAD}^{+a}$ dependent deacetylases, also referred to as type III histone deacetylases, that modify the acetylation state of several intracellular messengers, thereby regulating downstream mechanisms associated with these substrates. ${ }^{1}$ These deacetylases are able to integrate environmental cues and modulate several important physiological mechanisms such as inflammation, apoptosis, glucose homeostasis, life span, and neuroprotection. ${ }^{2}$ The pharmacological manipulation of theses enzymes has therefore strong potential for the therapy of many human diseases such as cancer, metabolic disorders, and degenerative diseases. ${ }^{3-5}$

Sirtuins (SIRT1-7 in mammals) are highly conserved enzymes. The most studied member of the sirtuin family is SIRT1. The SIRT1 protein is most related to the Caenorhabditis elegans sirtuin sir-2.1. Transgenic nematodes with increased sir-2.1 dosage can live up to $50 \%$ longer. ${ }^{6}$ Recent studies indicated that, depending on the cellular context and type of sirtuin considered, either the activation or the inhibition of sirtuins may protect against cell/tissue injury. ${ }^{7}$ Whereas sir-2.1/SIRT1 activation may protect against neuronal dysfunction in simple models of HD pathogenesis ${ }^{8}$ and models of ALS and A $\beta$ toxicity, ${ }^{9}$ the pharmacological inhibition of SIRT2 may be neuroprotective against $\alpha$-synuclein toxicity. ${ }^{10}$ Additionally, SIRT1 inhibition may alleviate

*To whom correspondence should be addressed. For C.N.: christian. neri@inserm.fr. For chemistry, A.M.: phone, +39 064991 3392; fax, +3906491 491; E-mail, antonello.mai@uniroma1.it.

${ }^{a}$ Abbreviations: ALS, amyotrophic lateral sclerosis; APP, amyloid precusor protein; $\mathrm{A} \beta, \beta$-amyloid peptide; FoxO, forkhead box class $\mathrm{O}$; GFP, green fluorescent protein; HD, Huntington's disease; NAD ${ }^{+}$, nicotinamide adenine dinucleotide; NAM, nicotinamide; OPMD, oculopharyngeal muscular dystrophy; PABPN1, polyadenylate-binding protein, nuclear, 1; PS1, presenilin-1 transmembrane protein; RNAi, RNA interference; RT-PCR, reverse transcription-polymerase chain reaction. gene silencing in Fragile $\mathrm{X}$ mental retardation syndrome under treatment with nicotinamide (NAM) or splitomicin (7, Figure 1). ${ }^{11}$ Sirtuin inhibition may also be involved in the beneficial effect of NAM observed in the triple-transgenic model of Alzheimer's disease harboring PS1, APP, and tau mutations. ${ }^{12}$ Finally, the genetic (loss-of-function; LOF) and pharmacological (sirtinol 1, Figure 1) inhibition of sir-2.1/ SIRT1 protects $C$. elegans transgenics from the toxicity of polyalanine expansion in the oculopharyngeal muscular dystrophy (OPMD) protein PABPN1, an effect that requires the transcriptional factor and key longevity protein daf-16/ FoxO,${ }^{13}$ suggesting that sirtuin inhibitors may be protective in OPMD through cell survival mechanisms.

Before evaluation of sirtuin modulators in mouse models of disease, it is important to identify potent lead compounds and to test whether they may be active in physiological conditions. Because PABPN1 nematodes show cellular (progressive loss of muscle cell nuclei) and behavioral (defective motility) phenotypes amenable to drug screening, ${ }^{13}$ we hypothesized that these animals may allow to select for sirtuin inhibitors that best rescue the toxicity of mutant PABPN1 at the muscle cell and behavioral levels.

In this study, we tested some known SIRT inhibitors, such as 1, ${ }^{14,15}$ meta-sirtinol 2, ${ }^{15}$ para-sirtinol 3, ${ }^{15}$ salermide $\mathbf{4},{ }^{16} \mathbf{7},{ }^{17} \mathrm{EX}-527 \mathbf{1 1},{ }^{18}$ and AGK-2 12, ${ }^{10}$ prepared by us according to published procedures (Figure 1). In addition, we tested two new synthetic sirtinol analogues (5 and $\mathbf{6})$, a splitomicin derivative (the ketosplitomicin analogue 8), and the benzodeazaoxaflavines (BDF4s) 9 and 10, two totally different compounds recently identified in our lab (Figure 1). Compounds $\mathbf{1 - 1 2}$ were tested for their ability to inhibit the enzymatic activity of SIRT1 and SIRT2 in vitro and to modulate mutant PABPN1 toxicity in transgenic nematodes.

Published on Web 12/30/2009 


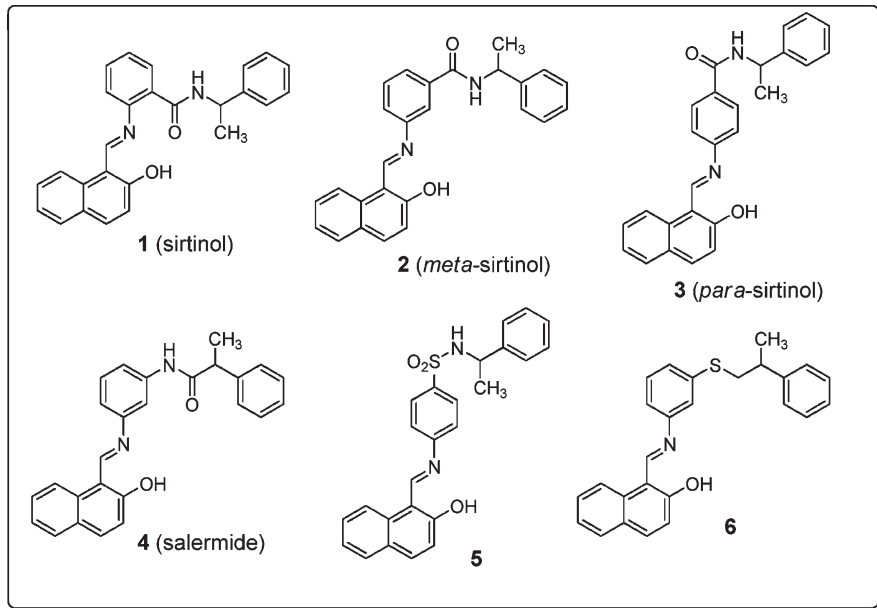

sirtinol and its analogues

Figure 1. Structures of sirtuin inhibitors described in this study.

\section{Chemistry}

The syntheses of $\mathbf{1}-\mathbf{4}$ were previously reported by us. ${ }^{15,16}$ Reaction between 4-nitrobenzenesulfonyl chloride and 1-phenylethylamine in the presence of triethylamine $\left(\mathrm{Et}_{3} \mathrm{~N}\right)$ in dry dichloromethane furnished the 4-nitro$N$-(1-phenylethyl)benzenesulfonamide 13, which was in turn reduced with stannous chloride and $35 \%$ hydrochloric acid $(\mathrm{HCl})$ in ethanol to the 4-amino analogue $\mathbf{1 4}$ and then condensed with 2-hydroxy-1-naphthaldheyde in the presence of glacial acetic acid to yield 5 (Scheme S1a in Supporting Information (SI)). The meta-thioether analogue of sirtinol, 6, was prepared by alkylation of 3-aminothiophenol with 1-bromo-2-phenylpropane in dry $\mathrm{N}, \mathrm{N}$-dimethylformamide (DMF) in the presence of potassium carbonate, followed by condensation of the obtained intermediate 15 with 2-hydroxy-1-naphthaldheyde in acidic medium (Scheme S1b in SI).

A 7 sample was synthesized according to the literature, ${ }^{19}$ while the ketosplitomicin derivative $\mathbf{8}$ was obtained by reaction of ethyl 3-(2-chlorophenyl)-3-oxopropanoate ${ }^{20}$ and 2-hydroxy-1-naphthaldheyde in dry ethanol with catalytic amounts of piperidine and glacial acetic acid. The 2-(2-chlorobenzoyl)-3H-benzo[f]chromen-3-one, 16, thus obtained was reduced with sodium borohydride in dry pyridine to afford the $1 H$-benzo[f]chromen-3(2H)-one derivative 8 (Scheme S2 in SI).

The two BDF4 derivatives, $\mathbf{9}^{21}$ and $\mathbf{1 0},{ }^{22}$ already known in literature as organic oxidants, were both prepared by condensation of the appropriate barbituric acids and 2-chloro1 -arylaldheydes in ethanol in the presence of pyridine, as described by Chen et al. ${ }^{22}$ for the synthesis of $\mathbf{1 0}$ (see Scheme S3 in SI). Compound $\mathbf{1 1}$ was synthesized as previously reported, ${ }^{18}$ while 12 was purchased from Sigma-Aldrich.

Experimental procedures, chemical and physical data (Table S1, SI), and elemental analyses (Table S2, SI) for compounds 5, 6, 8, 13-16 are reported in SI.

\section{Results and Discussion}

Adult $C$. elegans transgenics that specifically coexpress nuclear GFP and mutant PABPN1 (PABPN1-A13) in muscle cells show two major phenotypes including defective motility as reflected by an increased number of body bends, an effect accompanied by a loss of nuclear GFP signals indicative

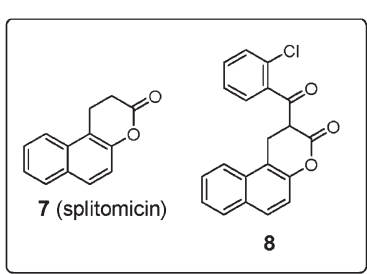

splitomicin and its analogue
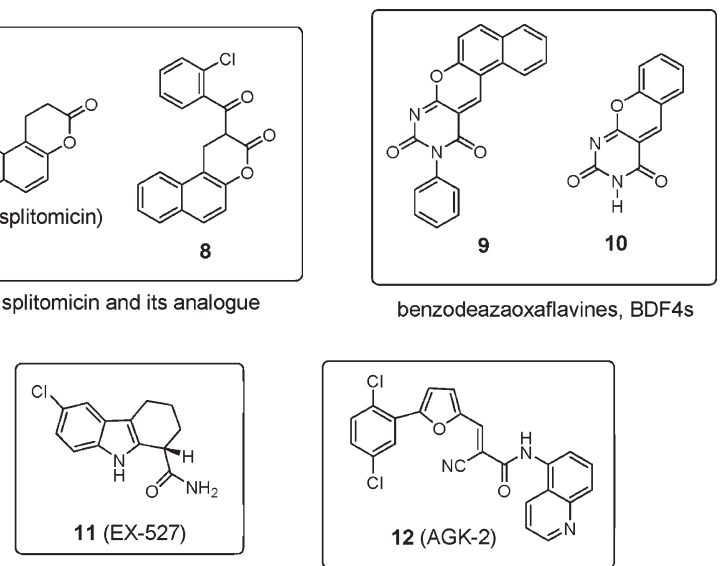

of a nuclear collapse. ${ }^{13}$ Consistent with the finding that sir-2.1/SIRT1 LOF is protective in these animals, with increased sir-2.1/SIRT1 dosage being detrimental, we previously reported that sir-2.1 RNAi rescues the loss of GFP signals in PABPN1A13 animals ${ }^{13}$ and that the sirtuin inhibitor $\mathbf{1}$ is able to rescue mutant PABPN1 toxicity in C. elegans muscle cells. ${ }^{13}$ However, 1 shows a moderate rescue of the loss of GFP signals $\left(\mathrm{ED}_{50}\right.$ above $1 \mu \mathrm{M}$ ) and defective motility (about $40 \%$ rescue compared to untreated animals) ${ }^{13}$ suggesting that it may be poorly effective in mouse models of OPMD pathogenesis. ${ }^{23}$

To search for more promising sirtuin inhibitors, we synthesized a series of sirtinol analogues ${ }^{15,16}$ by shifting the orthobenzamide moiety to the meta or the para position and/or by replacing the benzamide group with an anilide, sulfonamide, or thioether function (compounds 2-6, Figure 1), and we tested them for the inhibition of the enzymatic activity of SIRT1 and SIRT2 in comparison to 1 . We observed that all compounds were able to inhibit SIRT1 and SIRT2 (Figure 2A). Compared to $\mathbf{1}\left(\mathrm{IC}_{50} \mathrm{s:}: 123.3\right.$ and $45.9 \mu \mathrm{M}$ against SIRT1 and SIRT2, respectively), the $\mathbf{2 - 6}$ analogues were more efficient in inhibiting both SIRT1 and SIRT2 $\left(\mathrm{IC}_{50}\right.$ values: $40.3-58.6 \mu \mathrm{M}$ (SIRT1) and 19.2-34.5 $\mu \mathrm{M}$ (SIRT2)) (see SI). Upon evaluation in PABPN1 nematodes treated with $100-0.1 \mu \mathrm{M}$ concentrations from the $\mathrm{L} 1$ larvae stage until young adulthood, almost all the tested compounds were able to modulate the loss of nuclear GFP signals in mutant PABPN1-A13 nematodes, with no effect observed in normal PABPN1-A10 nematodes (Figure 2B). Of note, 6 did not show any effect on the loss of nuclear GFP signals (Figure 2B). This compound was thus not tested for effect on motility. The inability of $\mathbf{6}$ to reach muscle cells is likely to explain the lack of effect because all of the other related compounds were active in nematodes (Figure 2B). These effects were unrelated to a reduction in transgene expression as tested by RT-PCR and Western blotting (Figure S1, SI). However, rescue by $\mathbf{5}$ was accompanied by increased PABPN1 expression with no effect detected in RT-PCR (Figure S1, SI), suggesting that $\mathbf{5}$ may stabilize PABPN1. The para-substituted sirtinol analogue $\mathbf{3}$ enhanced mutant PABPN1 toxicity (Figure 2B), it likely being not specific of sir-2.1 (there are three other sirtuins in worms); on the other hand, the para-substituted benzenesulfonamide $\mathbf{5}$ was one of the most effective compounds in rescuing the loss of nuclear GFP signals (Figure 2B) and defective motility (Figure $2 \mathrm{C}$ ), with a $R_{\max }$ value above $10 \%$ and $\mathrm{EC}_{50}$ lower 

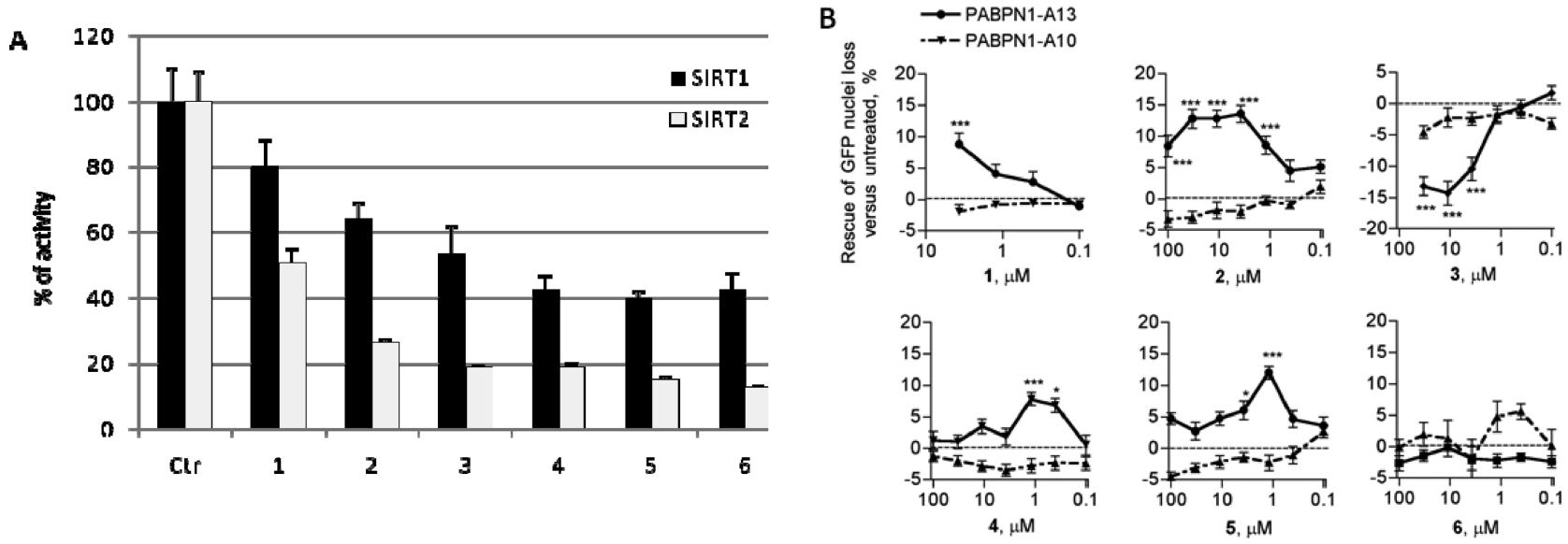
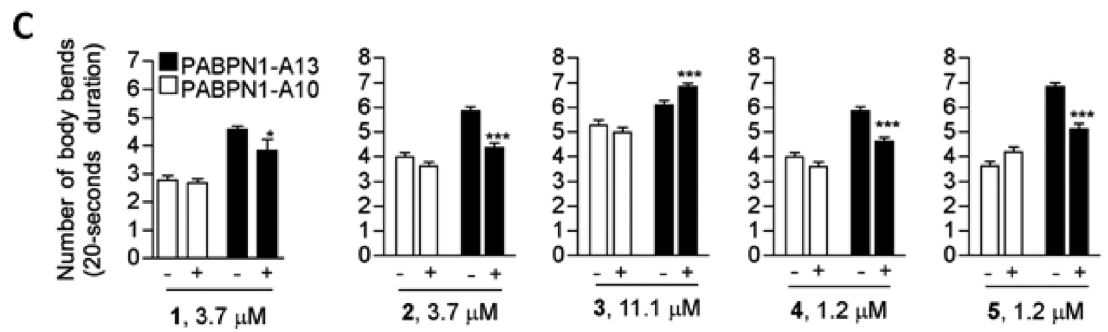

Figure 2. Characterization of sirtinol analogues: (A) SIRT1 and -2 inhibitory activity, expressed as \% of deacetylase activity. Compounds were tested at $50 \mu \mathrm{M}$. (B) Ability to rescue mutant PABPN1 (PABPN1-A13) toxicity (loss of nuclear GFP nuclei) in transgenic nematodes in comparison with that observed in normal PABPN1 (PABPN1-A10) nematodes. (C) Motility assay (number of body bends) in mutant and normal nematodes. Data are represented as mean $\pm \mathrm{SEM} * P<0.05$ and $* * * P<0.01$ compared to untreated animals (statistical effects were resistant to one-way ANOVA test).

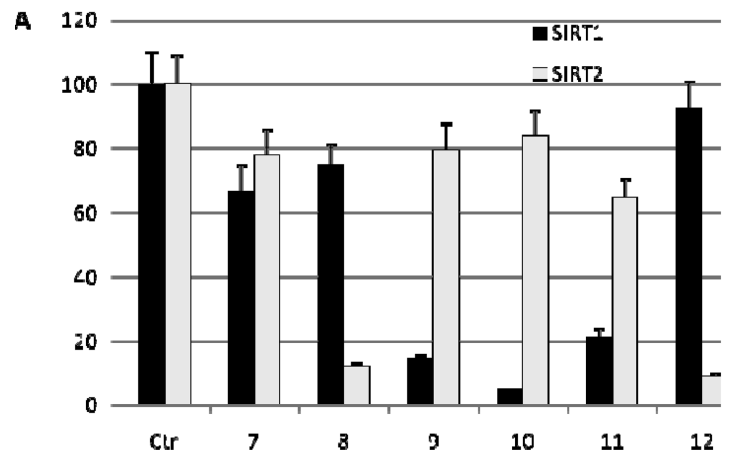

C

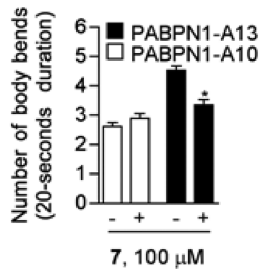

B
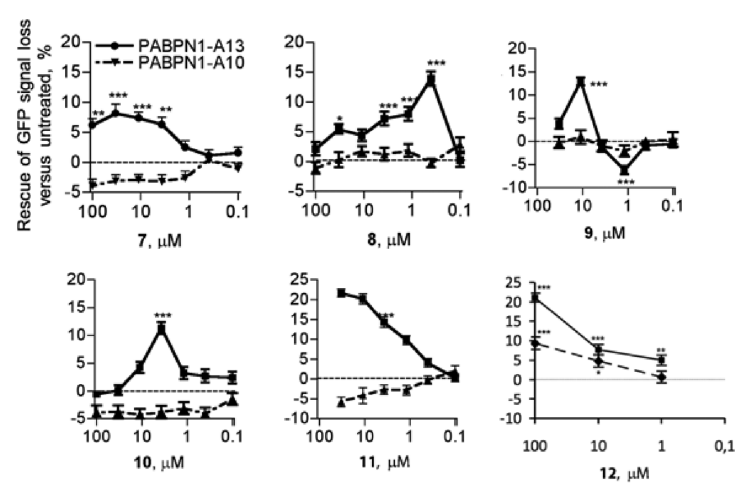

Figure 3. Characterization of 7-12: (A) SIRT1 and -2 inhibitory activity, expressed as \% of remaining deacetylase activity. Compounds were tested at $0.5(\mathbf{1 1}), 5(\mathbf{1 2})$, and 50 (others) $\mu \mathrm{M}$. (B) Ability to rescue mutant PABPN1 (PABPN1-A13) toxicity (loss of nuclear GFP nuclei) in transgenic nematodes in comparison with that observed in normal PABPN1 (PABPN1-A10) nematodes. (C) Motility assay (number of body bends) in mutant and normal nematodes. Data are represented as mean $\pm \mathrm{SEM} * P<0.05, * * P<0.01$ and $* * * P<0.001$ compared to untreated animals (statistical effects were resistant to one-way ANOVA test). For detail, see legend of Figure 2.

than $1 \mu \mathrm{M}$. This suggests that two parameters may influence the sirtinol analogues' efficiency in PABPN1 nematodes: (i) the position of the substituent at the benzimine portion, and (ii) the nature of the chemical link. The two metasubstituted sirtinol analogues $\mathbf{2}$ and $\mathbf{4}$ were the most efficient compounds for rescuing cellular phenotypes compared to $\mathbf{1}$, 
and they almost fully rescued defective motility back to the level of normal PABPN1 animals when tested in the $1-4 \mu \mathrm{M}$ range.

Next, we compared $\mathbf{7}$ with the ketosplitomicin analogue $\mathbf{8}$, carrying a 2-chlorobenzoyl moiety at the position 2 of the $1 H$-benzo $[f]$ chromen-3(2H)-one nucleus (Figure 1). 8 was found to strongly inhibit SIRT2 $\left(\mathrm{IC}_{50} \mathrm{SIRT2}_{2}=9.4 \mu \mathrm{M}\right)$, with a limited ability to inhibit SIRT1 $\left(\mathrm{IC}_{50}{ }^{\mathrm{SIRT} 1}=89.8 \mu \mathrm{M}\right)$ (Figure 3A). 7 was protective in mutant PABPN1 nematodes, showing a profile of activity (Figure $3 \mathrm{~B}, \mathrm{C}$ ) which is very similar to that of $\mathbf{1}$. Compared to $\mathbf{7 , 8}$ showed a greater ability to rescue mutant PABPN1 cytotoxicity in nematodes with no effect observed in normal PABPN1 animals (Figures 3B,C) and no reduction of mutant PABPN1 expression (Figure S1, SI). Thus, similarly to what was observed for sirtinol analogues, 8 provided a further example of the correlation between SIRT2 inhibition and rescue of mutant PABPN1 toxicity.

Afterward, we tested two novel SIRT inhibitors disclosed by us and bearing a benzodeazaoxaflavine (BDF4) structure, $\mathbf{9}$ and $\mathbf{1 0}$ (Figure 1). Compounds $\mathbf{9}$ and $\mathbf{1 0}$ were found to be two selective and highly potent SIRT1 inhibitors (9: $\mathrm{IC}_{50}$ SIRT1 $\left.=8.4 \mu \mathrm{M} ; 10: \mathrm{IC}_{50}{ }_{\mathrm{SIRT1}}=5.3 \mu \mathrm{M}\right)$, whereas they were much less efficient against SIRT2 (Figure 3A). 9 rescued the loss of nuclear GFP signals at high concentration $11.1 \mu \mathrm{M}$ and enhanced toxicity at low concentration $1.2 \mu \mathrm{M}$ (Figure 3B). The same was true for the modulation of defective motility (Figure 3C). In regard to 10, this compound showed at $3.7 \mu \mathrm{M}$ an efficient rescue of the loss of nuclear GFP signals (Figure 3B), and a moderate rescue of defective motility in mutant PABPN1 animals (Figure 3C). These effects were unrelated to a change in transgene expression (Figure S1, SI), and both compounds showed no effect in normal PABPN1 animals.

Finally, we tested in our C. elegans system 11 as a sample of potent, SIRT1-selective inhibitor, and $\mathbf{1 2}$, described as a SIRT2-selective inhibitor. Our enzyme assays performed on $\mathbf{1 1}$ and $\mathbf{1 2}$ gave the expected results in terms of SIRT1 and SIRT2 inhibition (Figure 3A). In normal (PABPN1-A10) and mutant (PABPN1-A13) PABPN1 nematodes, 11 rescued the loss of nuclear GFP signals also at low $\mu \mathrm{M}$ concentration (Figure 3B) and fully rescued defective motility at $33.3 \mu \mathrm{M}$ (Figure 3C). Compound 12, when tested on the loss of GFP nuclei, showed a significant and dose-dependent rescue (max $20 \%$ ) of loss of GFP nuclei as produced by mutant PABPN1 animals (Figure 3B). However, it also showed a statistically significant and dose-dependent increase (max $10 \%$ ) in the number of GFP nuclei of normal PABPN1 animals, thus its effect was not specific for mutant PABPN1 nematodes.

Altogether, the evaluation of compounds $1-12$ on SIRT $1 / 2$ inhibition in vitro and on modulation of mutant PABPN1 toxicity in transgenic nematodes indicated that (i) SIRT1/2 inhibitors such as $\mathbf{2}$ and $\mathbf{4}$ as well as the SIRT1-selective inhibitor $\mathbf{1 1}$ are highly potent rescuers of mutant PABPN1 toxicity, (ii) SIRT1-selective inhibitors such as $\mathbf{9}$ and $\mathbf{1 0}$ may also be active, however, showing a limited improvement of defective motility, and (iii) the SIRT2-selective inhibitor $\mathbf{1 2}$ show no specific effect for mutant PABPN1 animals.

\section{Conclusion}

The present study reveals chemical features that may support the rescue of mutant PABPN1 toxicity by sirtuin inhibitors in muscle cells. Motility is a strong criterion to discriminate compound activity in nematodes. The effect on muscle cell phenotypes provide another criterion to assess compound activity. In this respect, in the sirtinol analogue series the comparison of $\mathbf{1}$ to $\mathbf{2}, \mathbf{4}$, and $\mathbf{5}$ at chemical (compound structure), enzymatic (sirtuin inhibition), and biological (effects in PABPN1 animals) level strongly suggests that a better ability to protect from mutant PABPN1 toxicity correlates with (i) a meta-substituted benzamide or anilide moiety or para-substituted benzenesulfonamide moiety and (ii) an ability to inhibit both SIRT1 and SIRT2 in vitro. Other sirtuin inhibitors of interest raised by our study are 8, a ketosplitomycin analogue and highly active SIRT2 inhibitor, $\mathbf{1 0}$ and $\mathbf{1 1}$, two SIRT1-selective inhibitors, and 12, a SIRT2-selective inhibitor. Compared to the most effective sirtuin inhibitors described herein $(\mathbf{2}, \mathbf{4}$, and $\mathbf{1 1})$, compounds $\mathbf{8}$ and $\mathbf{1 0}$ showed a lower ability to rescue behavioral phenotypes in transgenic nematodes, and $\mathbf{1 2}$ displayed no specific effect in PABPN1 nematodes.

Future experiments will address the biochemical mechanisms underlying the protection from mutant PABPN1 toxicity in cell system by sirtuin inhibitors such as $\mathbf{2 , 4}$, and $\mathbf{1 1}$ and their therapeutic potential in mouse models of OPMD.

\section{Experimental Section}

SIRT Assay. Modulation of sirtuin activity by compounds was assessed using the Flour de Lys fluorescent biochemical assay available through BioMol International. In the first part of a two-step reaction, an acetylated lysine side chain present on the substrate was deacetylated during incubation at $37^{\circ} \mathrm{C}$ for $2 \mathrm{~h}$ with active enzyme (SIRT1 or SIRT2), compounds 1-12, and $\mathrm{NAD}^{+}$in white, 96-well polystyrene luminescence plates. The latter half of the reaction produced a fluorophore upon treatment with a developing reagent. The reaction was read by a fluorometric reader (Inphinite 200 TECAN) with excitation set at $360 \mathrm{~nm}$ and emission detection set at $460 \mathrm{~nm}$. Experiments on the SIRT1 and 2 inhibition were performed in quadruplicate.

Nematode Assay. $C$. elegans Strains. Transgenic nematodes expressing either the wild-type or the mutant form of PABPN1 protein were used as previously described. ${ }^{13}$

Drug Assays. For cellular assays, synchronized L1 animals were incubated in OP-50.1 liquid culture with the drug as previously described. ${ }^{8}$ The number of nuclear GFP signal was scored at the young adulthood stage using a $\times 10$ Zeiss fluorescence microscope (Axioplan Imaging II) as previously described. ${ }^{13}$ Percent rescue was calculated as ((test - control)/ $($ control $) \times 100)$. With this formula, a negative value means enhancement of PABPN1 toxicity, and the maximal achievable rescue is about $23 \%$. A minimum of 100 worms/test were scored per dose, and three independent assays performed. For motility assays, day 3-5 old adults were laid on a thin layer of OP-50.1 bacteria on NGM plates and the number of bodybends (complete sinusoïds) immediately measured for $20 \mathrm{~s}$ as previously described. ${ }^{13} \mathrm{~A}$ minimum of $60-100$ worms/test were scored per dose and three independent assays performed.

Statistical Analysis. Statistical analysis was performed using the two-tailed Student's $t$-test unless otherwise indicated in the legend of the figures.

Acknowledgment. We thank Bernard Brais for providing the human PABPN1 constructs, David Bear for providing the human PABPN1 antibody, and the Caenorhabditis Genetics Center for providing nematode strains. This work was supported by INSERM, FRM, the University of Paris Descartes, and the Association Française contre les Myopathies, Paris, France. M.Y.P. is supported by the University of Montreal. G.A.R. is supported by the Canadian Institutes of Health 
Research, the Muscular Dystrophy Association, and the Foundation of Greater Philadelphia. A.M. is supported by FIRB, RETI FIRB, and Fondazione Roma. L.A. is supported by AIRC; EU: "APO-SYS" HEALTH-F4-2007200767; "CancerDip" HEALTH-F2-2007-200620; "ATLAS” HEALTH-F4-2009-221952.

Supporting Information Available: Syntheses, chemical and physical data, and elemental analyses of compounds 5, 6, 8, and 13-16. PABPN1 transgene expression analysis, indicated by Q-RT PCR and Western blot analysis. This material is available free of charge via the Internet at http://pubs.acs.org.

\section{References}

(1) Westerheide, S. D.; Anckar, J.; Stevens, S. M., Jr.; Sistonen, L.; Morimoto, R. I. Stress-inducible regulation of heat shock factor 1 by the deacetylase SIRT1. Science 2009, 323, 1063-1066.

(2) Lavu, S.; Boss, O.; Elliott, P. J.; Lambert, P. D. Sirtuins-novel therapeutic targets to treat age-associated diseases. Nat. Rev. Drug Discovery 2008, 7, 841-853.

(3) Milne, J. C.; Lambert, P. D.; Schenk, S.; Carney, D. P.; Smith, J. J.; Gagne, D. J.; Jin, L.; Boss, O.; Perni, R. B.; Vu, C. B.; Bemis, J. E.; Xie, R.; Disch, J. S.; Ng, P. Y.; Nunes, J. J.; Lynch, A. V.; Yang, H.; Galonek, H.; Israelian, K.; Choy, W.; Iffland, A.; Lavu, S.; Medvedik, O.; Sinclair, D. A.; Olefsky, J. M.; Jirousek, M. R.; Elliott, P. J.; Westphal, C. H. Small molecule activators of SIRT1 as therapeutics for the treatment of type 2 diabetes. Nature 2007, $450,712-716$.

(4) Outeiro, T. F.; Marques, O.; Kazantsev, A. Therapeutic role of sirtuins in neurodegenerative disease. Biochim. Biophys. Acta 2008, 1782, 363-369.

(5) Taylor, D. M.; Maxwell, M. M.; Luthi-Carter, R.; Kazantsev, A. G. Biological and potential therapeutic roles of sirtuin deacetylases. Cell. Mol. Life Sci. 2008, 65, 4000-4018.

(6) Tissenbaum, H. A.; Guarente, L. Increased dosage of a sir-2 gene extends lifespan in Caenorhabditis elegans. Nature 2001, 410, 227 230

(7) Dillin, A.; Kelly, J. W. Medicine. The yin-yang of sirtuins. Science 2007, 317, 461-462.

(8) Parker, J. A.; Arango, M.; Abderrahmane, S.; Lambert, E.; Tourette, C.; Catoire, H.; Neri, C. Resveratrol rescues mutant polyglutamine cytotoxicity in nematode and mammalian neurons. Nat. Genet. 2005, 37, 349-350.

(9) Kim, D.; Nguyen, M. D.; Dobbin, M. M.; Fischer, A.; Sananbenesi, F.; Rodgers, J. T.; Delalle, I.; Baur, J. A.; Sui, G.; Armour, S. M.; Puigserver, P.; Sinclair, D. A.; Tsai, L. H. SIRT1 deacetylase protects against neurodegeneration in models for Alzheimer's disease and amyotrophic lateral sclerosis. EMBO J. 2007, 26, 31693179 .

(10) Outeiro, T. F.; Kontopoulos, E.; Altmann, S. M.; Kufareva, I.; Strathearn, K. E.; Amore, A. M.; Volk, C. B.; Maxwell, M. M.; Rochet, J. C.; McLean, P. J.; Young, A. B.; Abagyan, R.; Feany, M. B.; Hyman, B. T.; Kazantsev, A. G. Sirtuin 2 inhibitors rescue alpha-synuclein-mediated toxicity in models of Parkinson's disease. Science 2007, 317, 516-519.

(11) Biacsi, R.; Kumari, D.; Usdin, K. SIRT1 inhibition alleviates gene silencing in Fragile X mental retardation syndrome. PLoS Genet. 2008, 4, e1000017.

(12) Green, K. N.; Steffan, J. S.; Martinez-Coria, H.; Sun, X.; Schreiber, S. S.; Thompson, L. M.; LaFerla, F. M. Nicotinamide restores cognition in Alzheimer's disease transgenic mice via a mechanism involving sirtuin inhibition and selective reduction of Thr231phosphotau. J. Neurosci. 2008, 28, 11500-11510.

(13) Catoire, H.; Pasco, M. Y.; Abu-Baker, A.; Holbert, S.; Tourette, C.; Brais, B.; Rouleau, G. A.; Parker, J. A.; Neri, C. Sirtuin inhibition protects from the polyalanine muscular dystrophy protein PABPN1. Hum. Mol. Genet. 2008, 17, 2108-2117.

(14) Grozinger, C. M.; Chao, E. D.; Blackwell, H. E.; Moazed, D.; Schreiber, S. L. Identification of a class of small molecule inhibitors of the sirtuin family of NAD-dependent deacetylases by phenotypic screening. J. Biol. Chem. 2001, 276, 38837-38843.

(15) Mai, A.; Massa, S.; Lavu, S.; Pezzi, R.; Simeoni, S.; Ragno, R.; Mariotti, F. R.; Chiani, F.; Camilloni, G.; Sinclair, D. A. Design, synthesis, and biological evaluation of sirtinol analogues as class III histone/protein deacetylase (Sirtuin) inhibitors. J. Med. Chem. 2005, 48, 7789-7795.

(16) Lara, E.; Mai, A.; Calvanese, V.; Altucci, L.; Lopez-Nieva, P.; Martinez-Chantar, M. L.; Varela-Rey, M.; Rotili, D.; Nebbioso, A.; Ropero, S.; Montoya, G.; Oyarzabal, J.; Velasco, S.; Serrano, M.; Witt, M.; Villar-Garea, A.; Imhof, A.; Mato, J. M.; Esteller, M.; Fraga, M. F. Salermide, a Sirtuin inhibitor with a strong cancer-specific proapoptotic effect. Oncogene 2009, 28, 781-791.

(17) Bedalov, A.; Gatbonton, T.; Irvine, W. P.; Gottschling, D. E.; Simon, J. A. Identification of a small molecule inhibitor of Sir2p. Proc. Natl. Acad. Sci. U.S.A 2001, 98, 15113-15118.

(18) Napper, A. D.; Hixon, J.; McDonagh, T.; Keavey, K.; Pons, J. F.; Barker, J.; Yau, W. T.; Amouzegh, P.; Flegg, A.; Hamelin, E.; Thomas, R. J.; Kates, M.; Jones, S.; Navia, M. A.; Saunders, J. O.; DiStefano, P. S.; Curtis, R. Discovery of indoles as potent and selective inhibitors of the deacetylase SIRT1. J. Med. Chem. 2005, $48,8045-8054$.

(19) Posakony, J.; Hirao, M.; Stevens, S.; Simon, J. A.; Bedalov, A. Inhibitors of Sir2: evaluation of splitomicin analogues. J. Med. Chem. 2004, 47, 2635-2644.

(20) Mai, A.; Artico, M.; Sbardella, G.; Massa, S.; Novellino, E.; Greco, G.; Loi, A. G.; Tramontano, E.; Marongiu, M. E.; La Colla, P. 5-Alkyl-2-(alkylthio)-6-(2,6-dihalophenylmethyl)-3,4-dihydropyrimidin-4(3H)-ones: novel potent and selective dihydro-alkoxybenzyl-oxopyrimidine derivatives. J. Med. Chem. 1999, 42, 619627.

(21) Ridi, M.; Giovannelli, A. Barbituric acid and its derivatives. $\mathrm{X}$. Syntheses of pyrimidonaphtopyranic and naphthacoumarin derivatives. Gazz. Chim. Ital. 1952, 82, 13-22.

(22) Chen, X.; Tanaka, K.; Yoneda, F. Simple new method for the synthesis of 5-deaza-10-oxaflavin, a potential organic oxidant. Chem. Pharm. Bull. 1990, 38, 307-311.

(23) Davies, J. E.; Wang, L.; Garcia-Oroz, L.; Cook, L. J.; Vacher, C.; O'Donovan, D. G.; Rubinsztein, D. C. Doxycycline attenuates and delays toxicity of the oculopharyngeal muscular dystrophy mutation in transgenic mice. Nat. Med. 2005, 11, 672-677. 